\title{
Green Femtocell Based on UWB Technologies
}

\author{
Moshe $\operatorname{Ran}^{1}$ and Yossef Ben Ezra ${ }^{2}$ \\ ${ }^{1}$ MostlyTek Ltd. 58 Keshet St., Reut \\ ${ }^{2}$ H.I.T - Holon Institute of Technology, Holon, \\ Israel
}

\section{Introduction}

The rapid evolution of mobile communications through four generations of mobile communication, envisages the operation at $100 \mathrm{Mb} / \mathrm{s}$ for mobile users and at $1 \mathrm{~Gb} / \mathrm{s}$ for stationary applications in the near future. The tremendous increase of data rates must be considered in the context of four decades of the mobile cellular technologies progress since its first introduction by the Nippon Telephone and Telegraph Company (NTT) in the late 70's Rappaport (2002). On the other hand, fixed wireless communications are already available to provide over $300 \mathrm{Mbps}$ raw data rates through wireless local area networks (LAN) protocols as 802.11n, and over 1Gbps through Ultra Wideband (UWB) in wireless personal area networks (PAN), see (ECMA-368), (ECMA-387).

With the introduction of the femtocell concept Zhang (2010), new opportunities have been opened for approaching the $4 \mathrm{G}$ mobile vision through fixed mobile convergence (FMC). Femtocell Access Point (FAP), are low power access points that connect mobile terminal to the mobile core network using wired broadband or fixed broadband wireless technologies. The FAP provides viable opportunities for mobile operators, to meet the indoor coverage challenges for most demanding applications at low cost.

We propose a novel concept of $4 \mathrm{G}$ femtocell, denoted a "Green Femtocell", and high level network architecture to support the new paradigm of FMC, in which convergence of $4 \mathrm{G}$ cellular with short-range wireless and wired are realized. The proposed approach paves the way of green framework in which increase by $x 100$ in energy efficiency and x100 reduction of human exposure to wireless radiation become feasible.

Our approach relies on radio-over-fiber and all-optical solutions that can already be considered "green" in offering reduced energy consumption to alternative wireless access solutions, see CELTIC Purple Book (2011). The new concept is based on the following novel technical and business entities (Fig. 1):

- We introduce a green remote Home Access Node (HAN) that relays range of radio protocols, including UWB, WLAN, LTE-A, and IEEE $802.16 \mathrm{~m}$ as radio signals over hybrid wireless-fiber media from $1.8 \mathrm{GHz}$ to $10.6 \mathrm{GHz}$; with strict limitation of radiated power. Wireless radiation for indoor environments is reduced by 2-3 order of magnitudes, while potentially support target $1 \mathrm{Gbps}$ end-user data rates, by using dual-mode cellular-UWB for most common indoor applications. Indoor HAN should support mobile users at distances ranging from $0.3 \mathrm{~m}$ to $30 \mathrm{~m}$ over-the-air. For outdoor 
and longer range indoor topologies, we enable protocol-transparent architectures capable of relaying range of radio protocols from 30 to $300 \mathrm{~m}$.

- Processing for multiple HANs is centralized with optical Multi-cell Base Station (OMcBS) capable of performing parallel multiple input multiple output (MIMO) processing of radio-over-fiber (ROF) links over 100's of GRANs. Unlike the McBS approaches suggested in Foshini (2006) and Gambini (2010), our O-McBS approach involved with optical MIMO over multi mode fiber (MMF). The multicell processing performed at McBS enables clear benefits of centralized approach to interference management over the hybrid wireless-fiber medium and efficient radio resource managements (RRM). We note that MIMO over MMF is a very recent enabling technology that has been shown to attain $400 \mathrm{~Gb} / \mathrm{s}$ signalling rate over several hundreds of meters of MMF at 10-10 BER Greenberg (2007). A promising solution for radio signalling and multiple access over hybrid wireless-MMF is based on orthogonal frequency-division multiple access (OFDMA). However, most of the works have addressed only the indoor wireless channels Perez (2009).

- $\quad$ O-McBS are connected through optical femto gate way (O-FemtoGW) to core network through $\mathrm{Tb}$ /s optical links. O-FemtoGW multiplex data from 10's of O-McBS, and forms through all-optical real-time processing an optical OFDM (O-OFDM) signal carrying $100 \mathrm{~Gb} / \mathrm{s}$. Recently, works on all optical FFT schemes to implement efficiently O-OFDM to enable $1 \mathrm{~Tb} / \mathrm{s}$ have been published by Hillerkuss (2010). Recent survey on O-OFDM with MIMO can be found in Shieh (2010), and general aspects of O-OFDM in Armstrong (2009) and Gidding (2009).

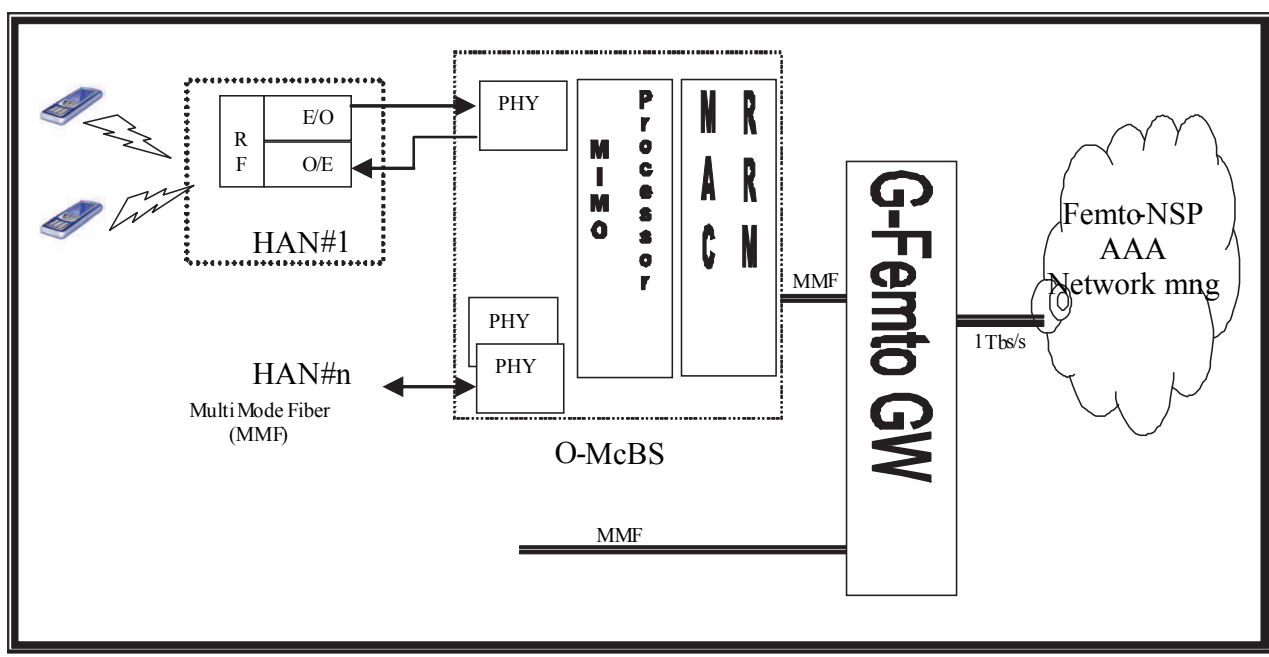

NSP: Network service provider O-McBS: optical multicell BS

MAC: Medium access control O/E: Optical/Electrical converter
MMF: Multimode Fiber

RRM: radio resource management

E/O: Electrical/Optical converter

Fig. 1. Green Femtocell Access Network (high-level architecture). 
The proposed architecture leverages and extends the concepts and technologies of UWB radio over optical fiber (UROOF) Ran (2010a, b), Ben-Ezra (2010), and further investigated in the context of future mobile technologies in Ran (2009) and Altman (2010). Our technical approach is directed to solve the crucial problem of interference management in local area environments in femtocells deployment. The femto-to-macro co-channel interference is solved by allocating unlicensed frequencies for indoor (e.g., UWB, WLAN). The femto-tofemto interference is addressed through centralized approach within the O-McBS.

In this chapter we focus on the local area coverage through HAN and elaborate possible indoor and outdoor architectures that can support $4 \mathrm{G}$ femto mobile vision in the near future. The chapter is organized into the following sections. In Section 2, we review state of the art of femtocell technology. In section 3, we review the very-low radiation distributed antenna system (VLR-DAS) concept. We elaborate indoor architectures and performances of green femtocells, and further extend the discussion to outdoor architectures in section 4 . In section 5, we provide interference analysis for single cell and multiple cell scenarios and discuss mitigation techniques to enable co-existence with other systems. Theoretical and experimental investigation is provided in Section 6. Conclusions are presented in Section 7.

\section{Femtocells technologies}

The use of densely deployed many low-power, low cost and high performance base stations, e.g., FAPs, seems a promising approach to cope with the ever-growing indoor coverage demand. Since $70 \%$ of voice and more than $90 \%$ of data services occur indoors Roche (2010), it becomes promising to deploy in-house FAP, which connects standard mobile devices to a mobile operator's through existing broadband Internet connection. According to a recent market prediction, Jarich (2010), leading femtocells vendors expect 2013 to be the year in which LTE and possibly WiMax femtocells will be commercially deployed. Femtocells are potentially industry-changing disruptive shift in technology for radio access in cellular networks. Femtocells should provide small scale functions of BS, and also certain functions of the customer premises equipment (CPE). Therefore, to gain benefits for network operators and consumer, the interaction between femto and macro radio layers should be carefully managed.

\subsection{State of the art of femtocells}

FAP's can be classified into two categories: home FAP, also called home base station (HBS), or enterprise FAP. HBS typically supports 2- 8 simultaneous users, where enterprise FAP can support 4 - 16 users, and can easily configured in cascade architecture to support 32- or 64-simultaneous users. FAP can further be classified according their underlined cellular technologies: GSM -, UMTS -, LTE-, WiMax -, FAP etc.

Femtocells can be configured in three ways to restrict and control their usage by certain users. In open access (or public access) all users, including outdoor users, or neighbouring femtocells are able to make use of nearby femtocells. In closed access (or private access) only a list of registered users are allowed to access a femtocell. In Hybrid access nonsubscribers use only limited amount of the femtocell resources, as e.g., emergency call services.

Open access benefits outdoor users, who are making use of nearby indoor femtocells and thus clearly improve the overall capacity of the network. From interference point of view, 
open access is superior to closed access, since it allows customers to connect to nearest access point. Thus it enables reducing the overall use of system resources (power-frequencytime). Possible drawback to this approach is the increase number of handoffs. Additionally, the femtocell customer pays by himself for the FAP and the broadband Internet connection, and is likely to reject the sharing of his own resources with users passing by his neighbourhood.

Some key technical challenges to large scale femtocell deployment are the following Yongho (2009):

- Interference to/from other femtocells and macrocell BS. Massive deployment will pose serious issues on the radio interference management with the surrounding cells (both femtocelss and macrocells). Since femtocells are planned to be installed in an ad-hoc manner by end-users and in large numbers, it will be challenging to do centralized and coordinated radio planning as in legacy macrocell system.

- Seamless Handover between a femtocell and macrocell or other femtocells. The conventional broadcast mechanism to advertise neighbour BS information may not be viable and scalable to include information about femtocells due to the excessive overhead needed. In the absence of this information, the macrocell-to-femtocell handover becomes challenging. In particular, handouts (femto to macro) and handin (macro to femto) efficient algorithms are required.

- Lack of standard solutions for scalability, redundancy and traffic partioning. For the femtocells to be widely used, it is essential that femto BSs interface with the rest of the network, both control and management planes, be fully standardised. No current guarantee that the fixed broadband connection will prioritize the traffic originating from the FAPs for a service without call blocking or dropping. It is highly desired that implementation of standard solutions be verified for multivendor interoperability.

- Synchronization and location. Inter-cell synchronization and femtocell location are critical for proper operation of femtocells, but GPS cannot be used in many indoor cases. Therefore, solutions for timing synchronization and location are needed.

Many aspects of current state of the art of femtocells technologies were published within the special issues of IEEE communications magazine (September 2009 and January 2010). Several research projects within the seventh EU framework program for research and technological development (FP7), and industry-driven research initiative CELTIC-Plus framework (www.celtic-initiative.org ) are addressing some key aspects of future femtocells technologies.

HOMESNET project, supported in part by CELTIC (CP6-009), is focused on three key challenges in HBS: dense deployment of self-organizing networks (SON), self-optimization and low radio emissions, Altman (2010). To achieve very low radio emissions in the house or constrained environment like hospitals, an architectural network option for HBS based on radio-over-fiber approach is investigated. The HOMESNET vision can certainly provide a good starting point for the new paradigm of Green Femtocell concept described in this chapter.

FREEDOM project (Femtocell-based Network Enhancement by Interference Management and Coordination, see www.ict-freedom.eu) aims at improving the efficiency of networks with massive femtocell deployment. The focus is on addressing the key question: How much the whole system efficiency can be improved by exploiting the available quality of the IP-based backhaul link? 
The solutions addressed in FREEDOM include the two main flavours of the 4G femtocell paradigm, namely IEEE $802.16 \mathrm{~m}$ and LTE-Advanced. In both cases the core concepts investigated are: Interference management and cooperation; dense femtocell-specific RRM; scalability and effectiveness and femtocell-based network planning.

BeFEMTO (Broadband Evolved FEMTO Networks, see www.ict-befemto.eu) is a recent integrated FP-7 project aiming to develop femtocell technologies based on LTE-A. The project is targeting ambitious objectives such as:

- Minimum system spectral efficiency of $8 \mathrm{~b} / \mathrm{s} / \mathrm{Hz} / \mathrm{cell}$.

- A maximum averaged transmit power of less than $10 \mathrm{~mW}$ for indoor femto nodes.

- Seamless convergence between fixed broadband and mobile cellular systems.

Rocket (Reconfigurable OFDMA-based Cooperative Networks Enabled by Agile Spectrum Use, see www.ict-rocket.eu) is another FP7 project aimed at providing solutions for LTE-A and $802.16 \mathrm{~m}$ to reach data rates $100 \mathrm{Mbps}$ and peak throughput higher than 1Gbps. The technical approach is based on advanced opportunistic spectrum usage, multi-user cooperative transmissions and ultra-efficient MAC design.

\subsection{Standardization of Femtocells}

Since a femtocell is a small scale cellular BS, it transmits over RF bands using licensed spectrum granted by the appropriate government authority. This requires that mobile operator be responsible for the control of radio transmission in a strict way, and follows regulations and standards. Standardization is certainly important for femtocells market to reach massive deployment. There are several standard development organizations (SDOs) and non-SDO forums that play an important role in the standardization of femto technology.

\section{SDOs:}

3GPP (www.3GPP.org) was created in 1998. The 3rd Generation Partnership Project (3GPP) unites 6 telecommunications standards bodies from Asia, Europe and North America. Over 350 companies participate in 3GPP through their membership of one of the 6 partners. The scope of 3GPP is to produce Specifications for a Mobile System based on evolved GSM core networks and the radio access technologies that they support. The femtocell concept applies as modifications for $2 \mathrm{G} / 3 \mathrm{G} / 4 \mathrm{G}$ mobile cellular generations described below in TABLE 1 .

Recent success with the creation of LTE and Systems Architecture Evolution (SAE) Specifications has made 3GPP the focal point for Mobile Broadband systems and a genuine contender as point of convergence for future Specifications for mobile networks. The standardization is defined in series of Technical Specification (TS) and Technical Reports (TR). The work is done through several working groups RAN2-RAN4 and SA1-SA5.

\begin{tabular}{|l|l|l|}
\hline 3GPP Radio Interfaces & $\begin{array}{l}\text { 2G radio: GSM, GPRS, EDGE } \\
\text { 3G radio: WCDMA, SSPA, HSPA, } \\
\text { LTE }\end{array}$ & $\begin{array}{l}\text { Rel.99 } \\
\text { Rel.4 }-7 \\
\text { Rel. } 8 / 9 \\
\text { Rel. } 10\end{array}$ \\
\hline 3GPP Core Network & $\begin{array}{l}\text { 2G/3G: GSM core network } \\
\text { 3G/4G: Evolved packet Core (EPC) }\end{array}$ & Rel. 8 \\
\hline 3GPP Service layer & $\begin{array}{l}\text { GSM, IMS, Multimedia Telephony } \\
\text { (MMTEL), }\end{array}$ & Rel. 9 \\
\hline
\end{tabular}

Table 1. 3GPP key releases and areas in which Femtocell concepts apply 
Recent reference model for stage 2 UTRAN architecture for 3G Home NodeB (HNB) was finalized in 3GPP RAN3 within TS 25.467. The basic elements of Iu-h interface are given in Fig. 2. The HNB connects to the mobile core network through HNB-GW, which acts as a concentrator to aggregate large number of HNB's. The Iu-h interface goes through a security gateway (SeGW) to HNB-GW. The HNB Management System (HMS) is based on TR-069 family of standards and provides authentication of HNBs and access from HNB to HNBGW.

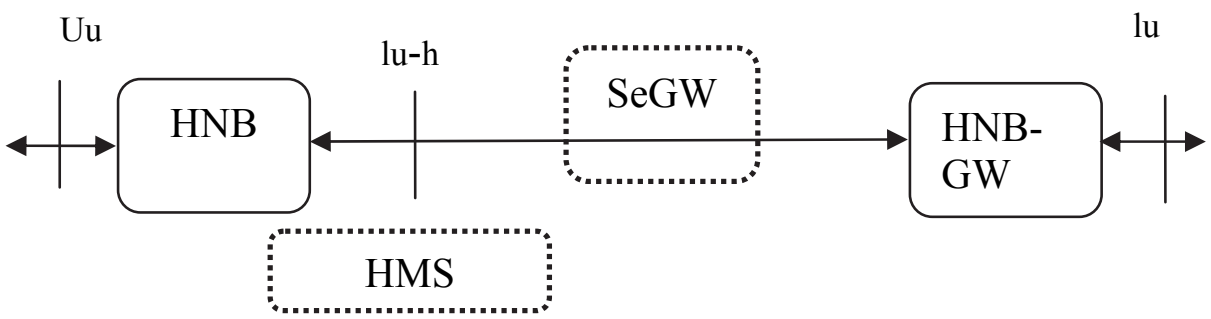

Fig. 2. Iu-h reference point in 3GPP for $3 \mathrm{G}$ Home NodeB.

Evolution to IMS/HSPA+/LTE is addressed in 3GPP TR R3.020. In January 2009, the 3GPP published overall architecture of LTE HeNB architecture within TS 36.300-870.

The $4 \mathrm{G}$ aspects of $3 \mathrm{GPP}$ are studied within the 3GPP approach to address the ITU's IMTadvanced system. Key $4 \mathrm{G}$ based on LTE-Advanced features, as defined within 3GPP Rel.10 and are summarized below.

- Channel BW. Support for wider bandwidth (up to $100 \mathrm{MHz}$ ).

- Downlink transmission scheme will support data rates of $100 \mathrm{Mb} / \mathrm{s}$ with high mobility and $1 \mathrm{~Gb} / \mathrm{s}$ with low mobility. It will be improvement to LTE along the evolution path by using 8x8 MIMO.

- Uplink transmission scheme supports data rates up to $500 \mathrm{Mb} / \mathrm{s}$.

- Relay functionality. Improvement to cell edge coverage and more efficient coverage in rural areas.

- Coordinated multiple point transmission and reception (CoMP) in both downlink and uplink

- $\quad$ LIPA (local IP Access) \& eHNB (enhanced HNB) to allow traffic off-load

3GPP2 (www.3GPP2.org) was created in 1999 as a partnership among SDOs from US, Korea and Japan and more recently from China to facilitate the CDMA based radio technologies for mobile cellular evolving from the IS-95 CDMA family of standards. The 3GPP2 architecture for femtocells is heading toward all-IP architecture for voice services based on Session Initiation Management Protocol (SIP), (see RFC3261), and IP multimedia subsystem (IMS) (see TS23.238)

BBF (Broadband Forum, previously DSL Forum, see www.brodbandforum.org) The TR-069 was originally created to manage DSL gateway device, and has been grown over the years for supporting new devices including femtocells. These modifications were published as amendments in (BBF TR-069), (BBF TR-098) and (BBF TR-106). In 2009 the forum published its data model for femtocells (BBF TR-196), supporting interoperability between FAP and network equipment. 
Non- SDO bodies and forums relevant to the femtocells paradigm:

The Femto Forum (www.femtoforum.org) has around 100 members from all parts of femtocell industry: major operators, major infrastructure vendors, vendors of components and subsystems. Femto Forum works with SDO and regulators worldwide to provide an aggregated view of femtocells market. The forum is focused on building and maintaining an eco-system that delivers the most commercial and technically efficient solutions based on femtocells. It has now four working groups (WGs): marketing \& promotion, radio \& physical layer, network \& interoperability and regulatory. Recently four special interest groups (SIGs) were founded: LTE, WiMAX, Interoperability and Services.

Femto Forum started discussions on femto architectures with 15 variations early 2008, and soon converged into only one based on Iu-h that has led to the proposal to 3GPP.

WiMAX forum (www.wimaxforum.org) is an industry-led organization that certifies and promotes the compatibility and interoperability of broadband wireless products based upon IEEE Standard 802.16. The forum has hundreds of members, comprising the majority of operators, component vendors and equipment vendors in the communications ecosystem. The WiMAX Forum's primary goal is to accelerate the adoption, deployment and expansion of WiMAX technologies across the globe while facilitating roaming agreements, sharing best practices within our membership and certifying products. WiMAX products are interoperable and support broadband fixed, nomadic, portable and mobile services. WiMAX has two phases for femtocell evolution:

Phase 1 is "femto aware" version based on IEEE802.16-Rev2 network release 1.6 and system profile release 1.0/1.5 with basically no change in the air interface standard to enable basic femtocell deployment. This version was completed late 2010.

Phase 2 "femto enhanced" version is based on network release 2.0; system profile release 2.0 and the air interface defined in $802.16 \mathrm{~m}$. This version is expected to be completed by 2012 with target deployments in 2012-2013.

NGMN (next Generation Mobile Networks http://www.ngmn.org) Alliance was founded by leading international mobile network operators in 2006, and joined recently (May 2011) the 3GPP as a market representation member. Its goal is to ensure that the standards for next generation network infrastructure, service platforms and devices will meet the requirements of operators and, ultimately, will satisfy end user demand and expectations.

GreenTouch (www.greentouch.org) is a recently established consortium dedicated to fundamentally transforming communications and data networks, including the Internet, and significantly reducing the carbon footprint of ICT devices, platforms and networks. By 2015, its goal is to deliver the architecture, specifications and roadmap - and demonstrate key components - needed to increase network energy efficiency by a factor of $\mathbf{1 0 0 0}$ from current levels.

\section{Green femtocell and Very-Low Radiation Distributed Antenna System (VLR-DAS)}

The basic idea of distributed antenna system (DAS) is replacing an antenna radiating at high power with $\mathrm{N}$ small antennas using low-power. Passive DAS use only passive elements as splitters, taps, terminators, circulators, filters and coaxial cables to split the RF signals into $\mathrm{N}$ antennas. Active DAS use different active elements (amplifiers, convertors E/O and O/E). Radio-over-fiber (ROF) DAS are most common active DAS techniques currently used. 


\subsection{Passive DAS}

The basic features of Passive DAS are well known Saleh (1987). The benefits of passive DAS using Omni antennas are discussed in Chow (1994). In particularly, the following useful features are evident:

- Maximizing coverage area. For a given radiated power, wireless channel with path loss exponent $\gamma, \mathbf{N}$ antennas system will have an increased coverage area over a single antenna system by factor $N^{1-\frac{2}{\gamma}}$.

- Minimizing the radiated power. For a given coverage area, an $\mathrm{N}$ antenna system will have a reduction in minimum required radiated power in downlink channel by the

factor of $N^{1-\frac{\gamma}{2}}$ compared to single antenna system. Furthermore, the maximum radiated power in the uplink channel will be reduced by the factor $N^{-\frac{\gamma}{2}}$.

- Minimizing maximum path loss. For a given coverage area, an $\mathrm{N}$ antenna system will have reduced maximum path loss by factor of $N^{-\frac{\gamma}{2}}$.

- Minimizing far-field interference. For a given coverage area, an N antenna system will have reduced far field interference by factor of $N^{-\frac{\gamma}{2}}$

Thus for example, for $\mathrm{N}=8$ and $\gamma=5$ and given coverage area, radiated power reduction of $22.6(13.5 \mathrm{~dB})$ will be achieved for the downlink and $181(22.6 \mathrm{~dB})$ in the uplink compared to a single antenna system. With such deployment, radiation from MS is reduced by $22.6 \mathrm{~dB}$ and thus the $\mathrm{C} / \mathrm{I}$ is improved by the same factor.

\subsection{Active DAS}

A typical active DAS uses master unit, which is the intelligent part of the active DAS, to distribute the RF signals from the BS antenna to multiple expansion units over an optical fiber of lengths up to $6 \mathrm{~km}$. Each expansion unit is connected to multiple remote radio units (RRUs) with thin coax, CAT5 or fiber of lengths up to $400 \mathrm{~m}$. Unlike passive DAS, active DAS has the ability to automatically compensate for the losses in the system by using internal calibrating signals and amplifiers. Active DAS is the preferred solution for large building and can provide monitoring and alarms in the event of malfunction.

DAS can be combined with MIMO communications concepts by treating the RRU's as a distributed antenna array, see Heath (2010). The multi-user MIMO DAS system model is given in Fig. 3

The model considers R RRUs in each cell, each BS and RRU are equipped with $\mathrm{N}_{t}$ antennas and mobile user with $\mathrm{N}_{\mathrm{r}}=1$ receive antennas. Some aspects of MIMO DAS using beamforming have been addressed in $\mathrm{Li}$ (2009). However, the overall benefits of multiuser MIMO DAS have not been established yet in DAS deployments Heath (2010).

Recently, there has been much research interest on the cooperative (or collocated) antenna system (CAS). It is shown You (2011) that theoretically CAS can alleviate major problems of the cellular systems as intercell interference (ICI) and cell edge effect problem. The performance of CAS depends very much on the degree of cooperation. In one extreme, the CAS consists of several distributed antennas which are connected to a central processing unit. This type of CAS is the DAS case, which has the best performance. In the other 
extreme, the BTS only exchange limited information, normally in order to boost the performance of the UEs at the edge of the cell. This case is also known as the Coordinated Multiple Point Transmission and Reception (CoMP) system. In this case, the transmission of the data is coordinated in time, frequency or space so as to minimize the inter-cell interferences and propagation loss effects. The CoMP concept for both uplink and downlink is central concept in LTE-A systems defined through 3GPP rel. 10 and future versions.

BS

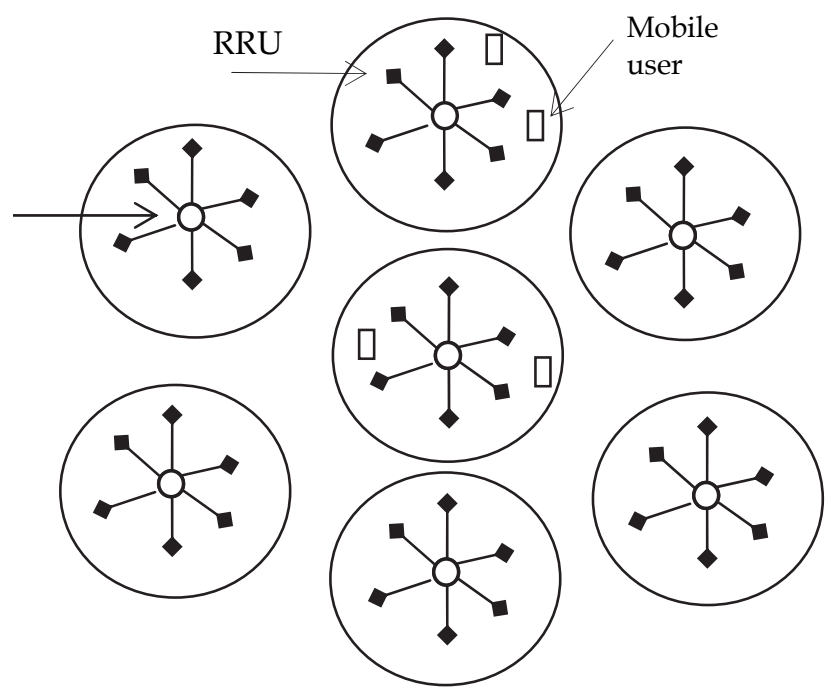

Fig. 3. The MIMO DAS multi-user system, Health (2010).

\subsection{Very Low Radiation DAS (VLR-DAS) approach}

Lowering energy consumption of future wireless radio systems along with great reduction of the wireless radiation indoor are important keys to next generation of mobile radio access systems. As transmitted data volume increases by factor 10 every 5 years, new technologies and solutions are becoming essential to support this trend while meeting the energy consumption along with the wireless radiation. The VLR-DAS concept, Ran (2010c), targets the distribution of various radio-protocols including 3G, LTE, IMT-Advanced and WiMax at a very-low radiation based on radio-over-X (optical fiber, Coax, Cat-5, power-line etc.). The focus is on scalable hybrid wired-wireless topologies to achieve a remarkable reduction of the emitted power to minimum level (order of $1 \mathrm{~mW}$ and less) needed for coverage of small cells.

VLR-DAS approach in the context of 3G femtocell paradigm is described in Fig. 4, Ran (2010c). The new functional device, called Home Access Node (HAN) is a special version of RRU with strict limitation of radiated power to below $1 \mathrm{~mW}$ over the air. HAN also used to adapt the RF signal to the wired media with minimum distortion, and perform initial identification and filtering of target radio signal. 


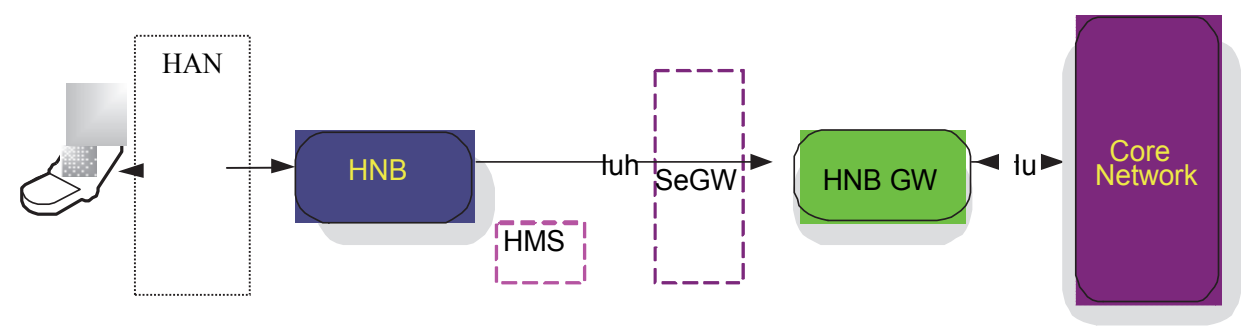

Fig. 4. 3G femtocell logical architecture Deployment configuration/Options with HAN function

Some general requirements for the successful operation of HAN with $3 \mathrm{G}$ femtocell are the following.

- The introduction of the HAN as an intermediate unit between HNB and the UE should not affect the $3 \mathrm{G}$ protocol stack between the HBS and the core network.

- Coexistence of the relayed radio signals with other radio signals (adjacent HBSs, macro BS, WLAN etc.) is required both for wired and for the wireless indoor infrastructure.

- The transmission of the various radio signals from the HBS through the HAN and the wired-wireless infrastructure to the UE should introduce minimum delay and negligible distortion to the performance of the communication link between HBS and UE.

\section{VLR-DAS basic scenarios}

Case 1: Residential in-house distribution network. For home users the VLR-DAS concept implies using the distribution of target wireless signals inside small number of rooms (say less than 8 ) based on existing and future home infrastructure. The radio signals are relayed transparently from one HBS to several rooms as "radio-over-wired" signals (with possibly frequency translate over wired home infrastructure). The wired part is serving as a "range extension" unit over 10's of meters to a set of HANs. Each HAN transmits at minimum power (less than $1 \mathrm{~mW}$ ) to cover a single room of less than $10 \mathrm{~m}$.

This case, shown in Fig. 5, is based on the simplified serial concatenation of several HAN's connected through a multimode fiber (MMF). The use of a separate fiber for the uplink and downlink conversion aimed to simplify and reduce the cost of the HAN implementation. In this example, HAN\#1 serves as the Master Unit (MU) that communicates with the HBS (or external macro BS either directly, or through a repeater) over the air to simplify the installation of the home network. The other HAN's transmit the radio signals over the air to the target UE.

Case 2: Enterprise "Green Hospital". The Green Hospital represents a corporate use case of citizen-to-authority and authority-to-citizen (C2A-A2C) where the exchange of large amount of medical data over $100 \mathrm{~s}$ of femtocells is considered. Here the range extension over the wired media is $100-3000 \mathrm{~m}$, and hundreds of users are supported through highly dense multi-femtocells architecture.

In hospitals the need for wireless is growing exponentially because all kinds of "vital signs" functions are monitored continuously. In hospital networks, there is an inherent inconsistency between the wish for mobility of the monitored patients and the interference of the radio signals with the sensitive diagnostic equipment. At present, some 8-10 functions per patient are wirelessly recorded. The number of monitored functions will grow rapidly in 
the coming years. In many cases the monitoring of patient functions is done wireless because of the need for improved "quality of life" for the patients. In essence, the use of "wireless" in a hospital is conflicting with the safety and security demands for the sensitive diagnostic and patient treatment equipment. Green Femtocell would be a great benefit by enabling unique capabilities to medical centres and health management offices (HMO).

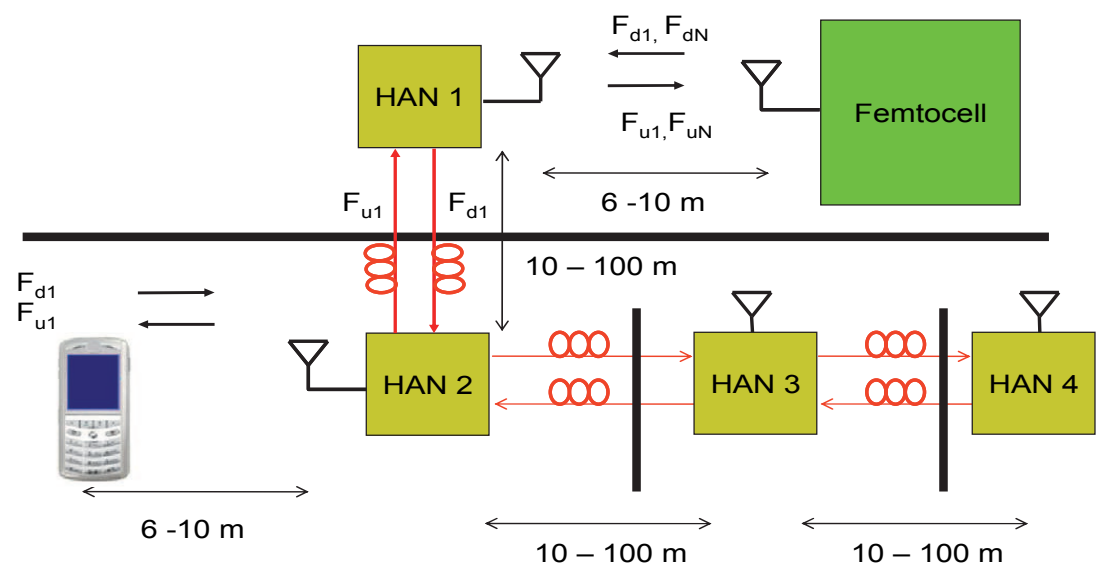

Fig. 5. Typical residential configuration of VLR-DAS over 4 rooms

Green hospital scenario for radiological department: The relevant patient data including previous examination images is transmitted within 1-2 seconds to the HAN sensor located near the desk. The data includes also medical information such as allergy to contrast media or renal failure, which will pop-up automatically on the screens of the staff in the department. The images are transferred from the HAN on the front desk immediately to the Picture Archiving and Communication System (PACS) via the wired optical fiber backbone. The PACS consists of Host Communication Controllers (HCCs) and the central Hospital Information System (HIS). HIS data would appear automatically on the computer screen on the desk. Then, the secretary has just to show the data to a visitor in order to get his confirmation that the data are correct.
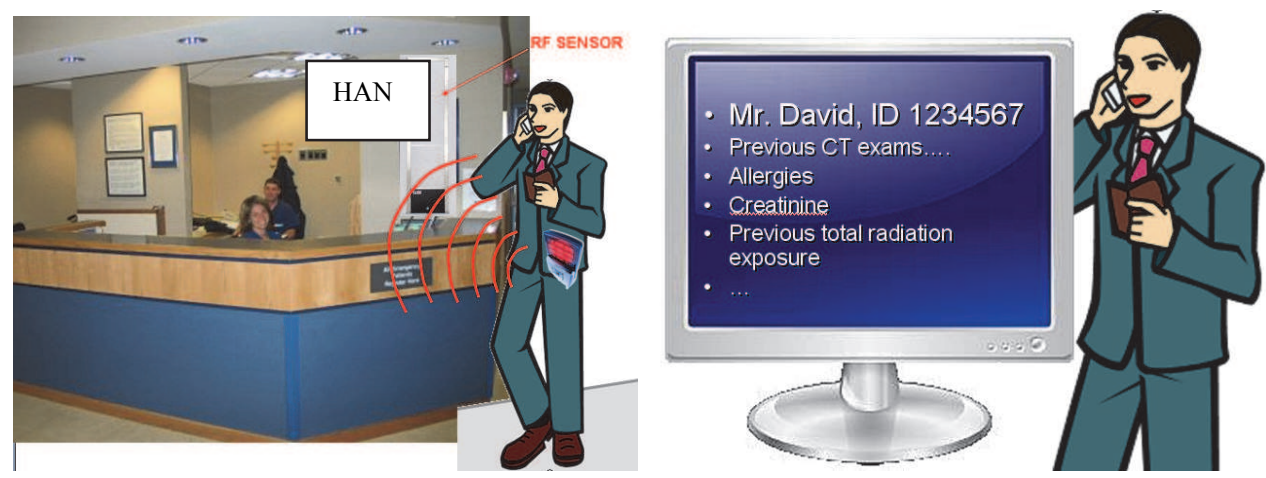

Fig. 6. Green Hospital scenario for radiological department. 
Derivatives of this scenario for fast exchange of medical records such as part/all the medical history of a patient may include: summary and reports of medical visits in different clinics, all laboratory results as blood tests, urine, previous ECG records, etc. obtained during the patient's life. It may include large files of diagnostic imaging procedures, pathology and histology results (including digitalized images), summary or lists of previous and currently active diseases, side effects and hypersensitivity reactions to drugs and contrast media, allergies, family history, a list of drugs taken by the patient, etc.

\subsection{Experimental results of VLR-DAS for 3G UMTS}

Some results of UMTS Green Femtocells were published in Ran (2010c). Fig. 7 shows the experimental setup for the testing of the VLR-DAS concept in Case 1. The system comprised a femto BS (Agilent E4432B) transmitting "green" W-CDMA FDD signals of -20dBm at band I (UL: $1920-1980 \mathrm{MHz}$ and DL: $2110-2170 \mathrm{MHz}$ ). We used the Test Model 1 containing 64 DPCH signal. The optical sub-system contained 10Gbps $850 \mathrm{~nm}$ Vertical-Cavity SurfaceEmitting Laser (VCSEL) for the direct E/O conversion of the W-CDMA signal into radioover-fiber (ROF) signal. The ROF UMTS signal propagated through a standard MMF (type OM3) of the length of $30 \mathrm{~m}$, and was detected by a PIN diode. Then, the detected signal was onward transmitted through the tested channel to the W-CDMA receiver. The purpose of this experiment was the study of the wired channel performance. The measured gain of the wired channel is about $3 \mathrm{~dB}$. The performance of the wired channel strongly depends on the RF signal power level at the VCSEL input due the VCSEL strong nonlinearity.

\section{$\mathrm{DL}-2.115 \mathrm{GHz}$}

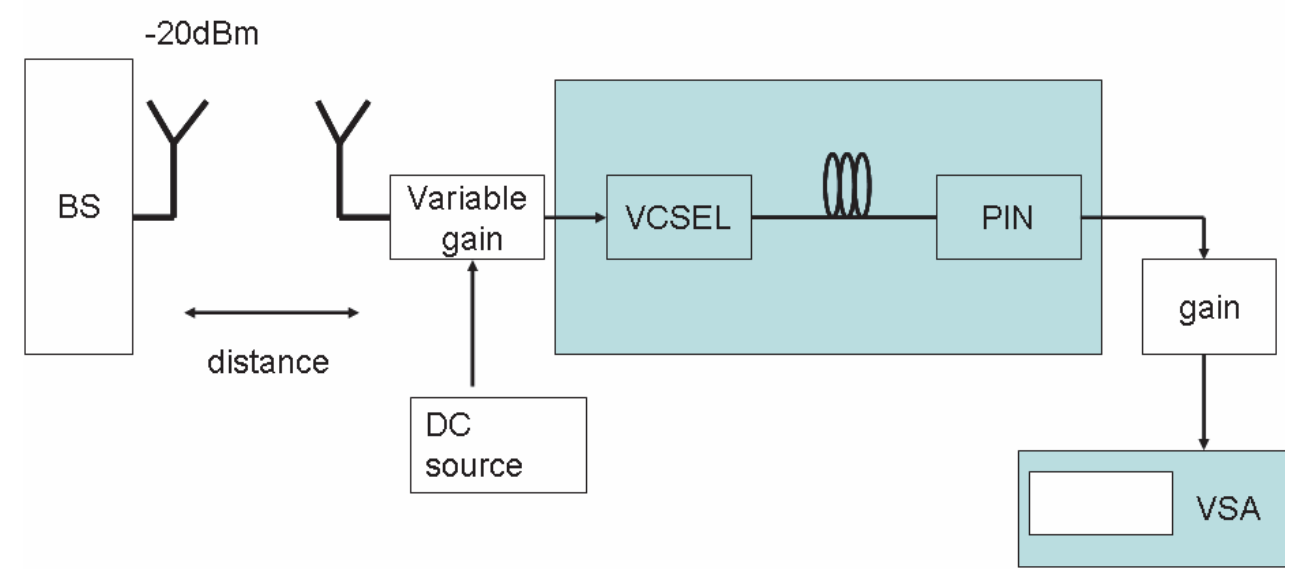

Fig. 7. Experimental set-up for UMTS in VLR-DAS scenario.

As shown in Fig. 8, the optimal value for the input RF power is about $-30 \mathrm{dBm}$. The input power values above $-25 \mathrm{dBm}$ lead to distortions expressed by higher order intermodulations and link gain degradation which affect the error vector magnitude (EVM). EVM and link gain vs. input signal power were recorded for the various scenarios. The EVM degradation, denoted $\triangle \mathrm{EVM}$, is obtained by comparing the EVM for the "best case" where BS is directly connected to the HAN, i.e., no optical or wireless segment, and the wired case where HAN is directly connected to the BS, and through the optical segment to the UE. 


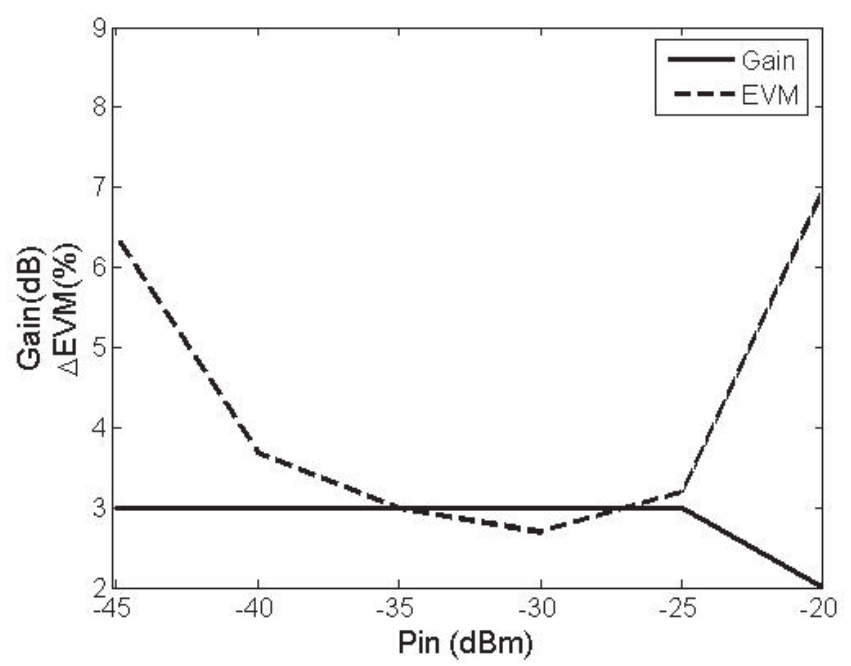

Fig. 8. $\triangle E V M$ and link gain for the wired case. The HAN is connected to the BS through a wired connection and to the user through VLR-DAS optical segment. The link shows negligible performance degradation over wide range of input power.

Fig. 9 shows the gain and $\triangle \mathrm{EVM}$ for a combined wireless-wired channel and different lengths of wireless segments. The measured gain of the combined channel is about $15 \mathrm{~dB}$. Although the combined wireless-wired channel causes the degradation of EVM in the range of $8 \%-15 \%$, still the satisfactory performance of EVM values below $11 \%$ is observed.

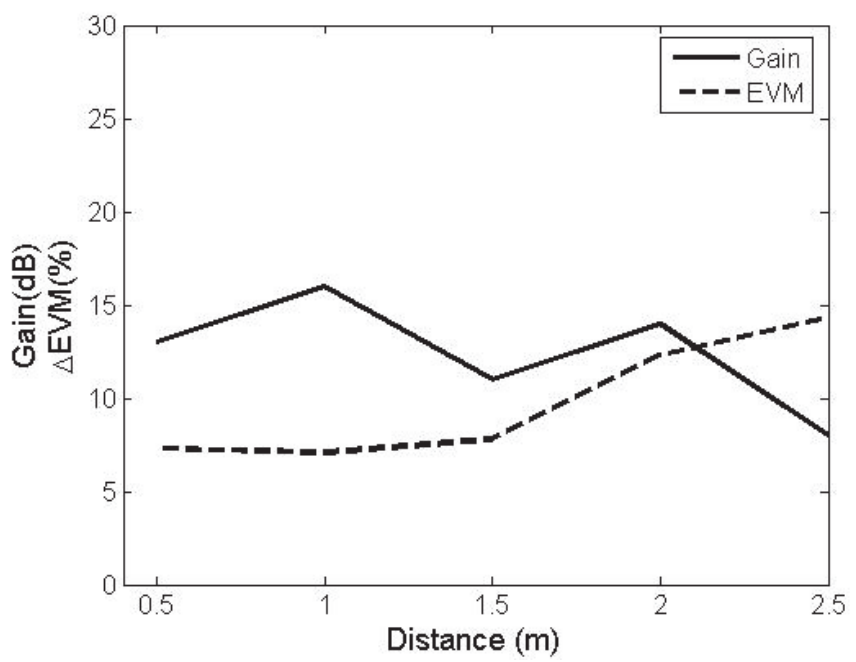

Fig. 9. $\triangle E V M$ and link gain for the wireless-wired case. The HAN is wirelessly connected to BS and connected to the user through VLR-DAS optical segment. 


\section{4G green femtocell: Indoor architectures based on MB-OFDM UROOF technologies}

The UWB radio over optical fiber (UROOF) was investigated recently in series of works, see (UROOF), Ran (2010a, b, c), Ben-Ezra (2010), Kraemer (2009) and Ran (2009). In this section we propose a framework, based on UROOF technologies, to elaborate indoor architectures for implementing the $4 \mathrm{G}$ green femtocell paradigm (see Fig. 1). We start with the proposed wireless air interface based on MB-OFDM UWB. We then provide some experimental data on transmitting multi-gigabits data over hybrid fiber-wireless channels and review MBOFDM with MIMO as a promising approach for the compound indoor wireless-MMF channel.

\subsection{MB-OFDM UWB over wireless indoor channel}

MB-OFDM has been proposed for the draft standard 802.15.3a (withdrawn on January 2006) and by the WiMedia Alliance in ECMA-368. MB-OFDM UWB is a combination of extremely broadband OFDM signal and frequency hopping, in which $528 \mathrm{MHz}$ channels are selected over the entire $7.5 \mathrm{GHz}$ bandwidth between 3.1 to $10.6 \mathrm{GHz}$. Time frequency codes (TFCs) of length 6 are used to select a sequence of "logical channels" from a band group. Unique logical channels are defined by using up to seven different TFC codes for each band group. TFCs for band group 1, according to (ECMA-368) are given for example in Table 2.

\begin{tabular}{|l|l|l|l|l|l|l|}
\hline TFC Number & \multicolumn{2}{|l}{ BAND_ID for Band Group 1 } & \multicolumn{2}{l|}{} \\
\hline 1 & 1 & 2 & 3 & 1 & 2 & 3 \\
\hline 2 & 1 & 3 & 2 & 1 & 3 & 2 \\
\hline 3 & 1 & 1 & 2 & 2 & 3 & 3 \\
\hline 4 & 1 & 1 & 3 & 3 & 2 & 2 \\
\hline 5 & 1 & 1 & 1 & 1 & 1 & 1 \\
\hline 6 & 2 & 2 & 2 & 2 & 2 & 2 \\
\hline 7 & 3 & 3 & 3 & 3 & 3 & 3 \\
\hline
\end{tabular}

Table 2. Time Frequency codes patterns for band group 1

There are 5 Band Groups (see Fig. 10):

- Band group \#1 is mandatory, remaining (\#2 - \#5) are optional.

- Only two Time-Frequency coded Logical Channels for Band group \#5.

Band group \#2 can be avoided when interference from U-NII bands is present.

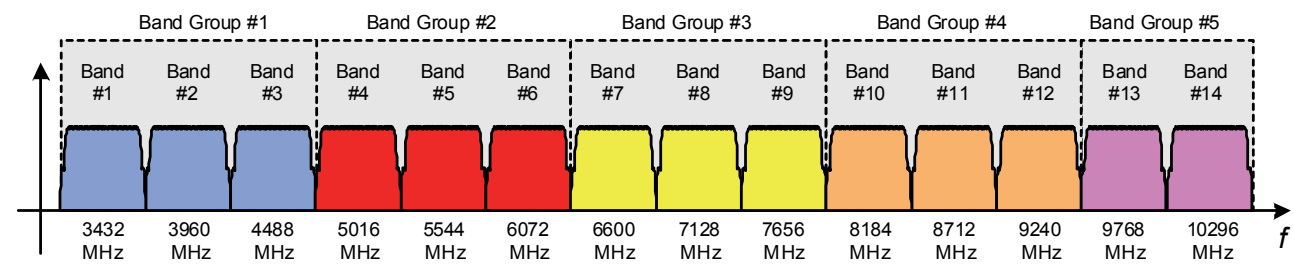

Fig. 10. Band plan for 3.1-10.6 GHz MB-OFDM with centre band frequencies shown. 
The MB-OFDM signal can be expressed by

$$
x_{M B-O F D M}(t)=\operatorname{Re}\left\{\sum_{k=0}^{N-1} x_{\text {OFDM }, k}\left(t-k T_{\text {OFDM }}\right) \exp \left[j 2 \pi f_{(k \bmod 6)} t\right]\right\}
$$

Where, $x_{O F D M, k}(t)$ is $k$ th the OFDM symbol of duration $T_{O F D M}, N$ is the number of transmitted OFDM symbols and $f_{k}$ is the carrier frequency over which the symbol is transmitted. The designed value for $T_{O F D M}$ is $312.5 \mathrm{~ns}$, where information length is $242.4 \mathrm{~ns}$, 9.5ns kept for guard time, and 60.6ns are length of the cyclic prefix, providing guard against multipath of length up to 60.6ns.

UWB wireless channel models were developed within IEEE 802.15.3a group during 2002 for the range $0-10 \mathrm{~m}$. The model defines four radio environments, based on simplified SalehValenzuela (S-V) channel model, Saleh (1987a):

CM1: near line-of-sight (LOS) with distance of 0-4 $\mathrm{m}$ between $\mathrm{Tx}$ and $\mathrm{Rx}$.

CM2, 3: non-LOS for a distance $0-4 \mathrm{~m}, 4-10 \mathrm{~m}$, respectively.

CM4: heavy multipath environment.

Realistic simulations over CM1-CM4 were carried out in which: losses due to front-end filtering, clipping at the DAC, DAC precision, ADC degradation, multi-path degradation, channel estimation, carrier tracking, packet acquisition, overlap and add of 32 samples (equivalent to $60.6 \mathrm{~ns}$ of multi-path protection), etc. were considered. The distance at which the MB-OFDM system can achieve a PER of $8 \%$ for a $90 \%$ link success probability is tabulated in Table 3 below:

\begin{tabular}{|c|c|c|c|c|c|}
\hline Range & AWGN & CM1 & CM2 & CM3 & CM4 \\
\hline $110 \mathrm{Mbps}$ & $20.5 \mathrm{~m}$ & $11.4 \mathrm{~m}$ & $10.7 \mathrm{~m}$ & $11.5 \mathrm{~m}$ & $10.9 \mathrm{~m}$ \\
\hline $200 \mathrm{Mbps}$ & $14.1 \mathrm{~m}$ & $6.9 \mathrm{~m}$ & $6.3 \mathrm{~m}$ & $6.8 \mathrm{~m}$ & $4.7 \mathrm{~m}$ \\
\hline $480 \mathrm{Mbps}$ & $7.8 \mathrm{~m}$ & $2.9 \mathrm{~m}$ & $2.6 \mathrm{~m}$ & N/A & N/A \\
\hline
\end{tabular}

Table 3. Simulation results of MB-OFDM over practical indoor channel models

An improved version to this model was published within IEEE 802.15.4a, based on more detailed field measurements. The model allows a larger number of environments; treats the number of clusters of multipath components in the $\mathrm{S}-\mathrm{V}$ as a random variable and allows frequency dependence of the path loss, according to the following formula.

$$
P L(f)=E\left\{\int_{f-\Delta f / 2}^{f+\Delta f / 2}\left|H\left(f^{\prime}\right)\right|^{2} d f^{\prime}\right\}
$$

$E\{\}$ is taken over large enough area to allow averaging out the small scale fading as well as shadowing. $\Delta f$ is chosen small enough so that the dielectric constant, diffraction coefficients are constant within that bandwidth. An updated survey on UWB propagation channels is given in Chapter 3 of Kraemer (2009). 


\subsection{MB-OFDM UWB radio over mixed wireless-MMF}

Channel impairments of MMF fibers are overviewed in Shieh (2010). When addressing the mixed wireless-MMF with MB-OFDM UWB technology, both frequency selective fading due to time delay spread over indoor channel, and multimode dispersion in MMF links should be considered. In particular, the cyclic prefix design for OFDM symbol should be longer than maximum delay due multipath wireless propagation and multimode dispersion spread in MMF.

A simplified model for UROOF "optical relay system" is given in Fig. 11, Ran (2010a), consisting of MMF, directly modulated VCSEL and a photodetector (PD) PIN diode.

It is shown based on series of experiments with UROOF platform (UROOF) that range extension by two orders of magnitude can be achieved for all MB-OFDM UWB RF signals.

A highly efficient method of RF and optical signal mixing to achieve optical OFDM transmission of MB-OFDM beyond 40Gbp/s was presented recently by Ben-Ezra (2010). The first concept is based on parallel-RF/serial optics architecture shown in Fig. 12. Basically, this architecture takes 128 conventional WiMedia/ECMA baseband channels of $528 \mathrm{MHz}$ and uses all-optical mixing to multiplex them over SMF. One of the key advantages of such approach is the ability to provide hybrid fiber-wireless solution, where the wireless segment at the available ultra-wideband (UWB) transmission is fully compliant with UWB regulations.

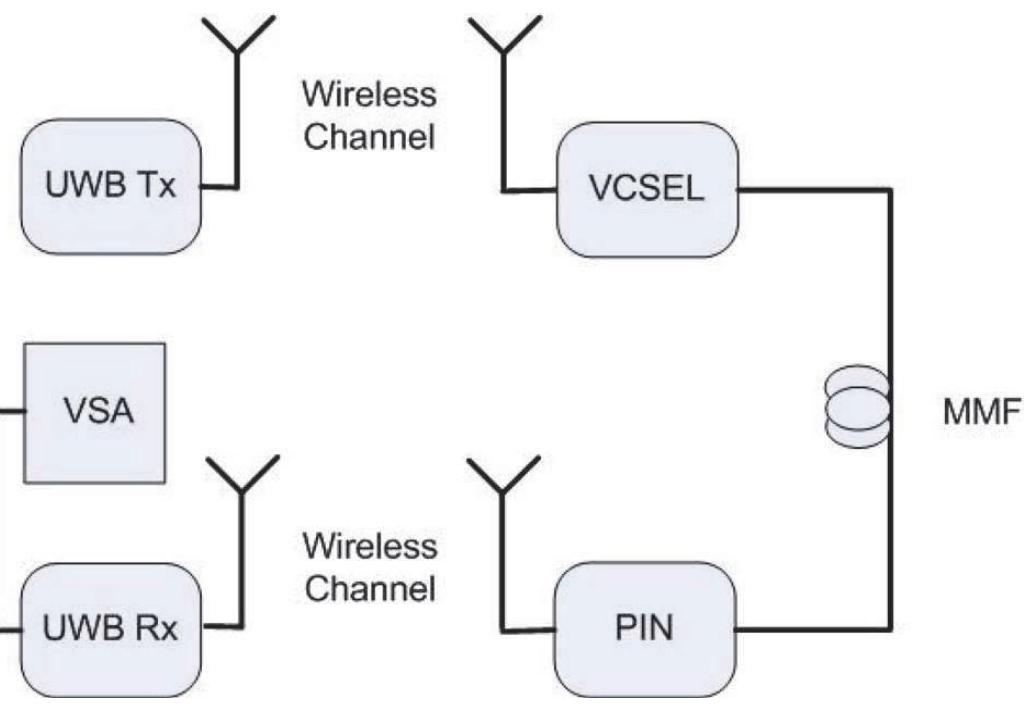

Fig. 11. UROOF channel consisting of an optical link (VCSEL-MMF-PD) and a wireless channel 


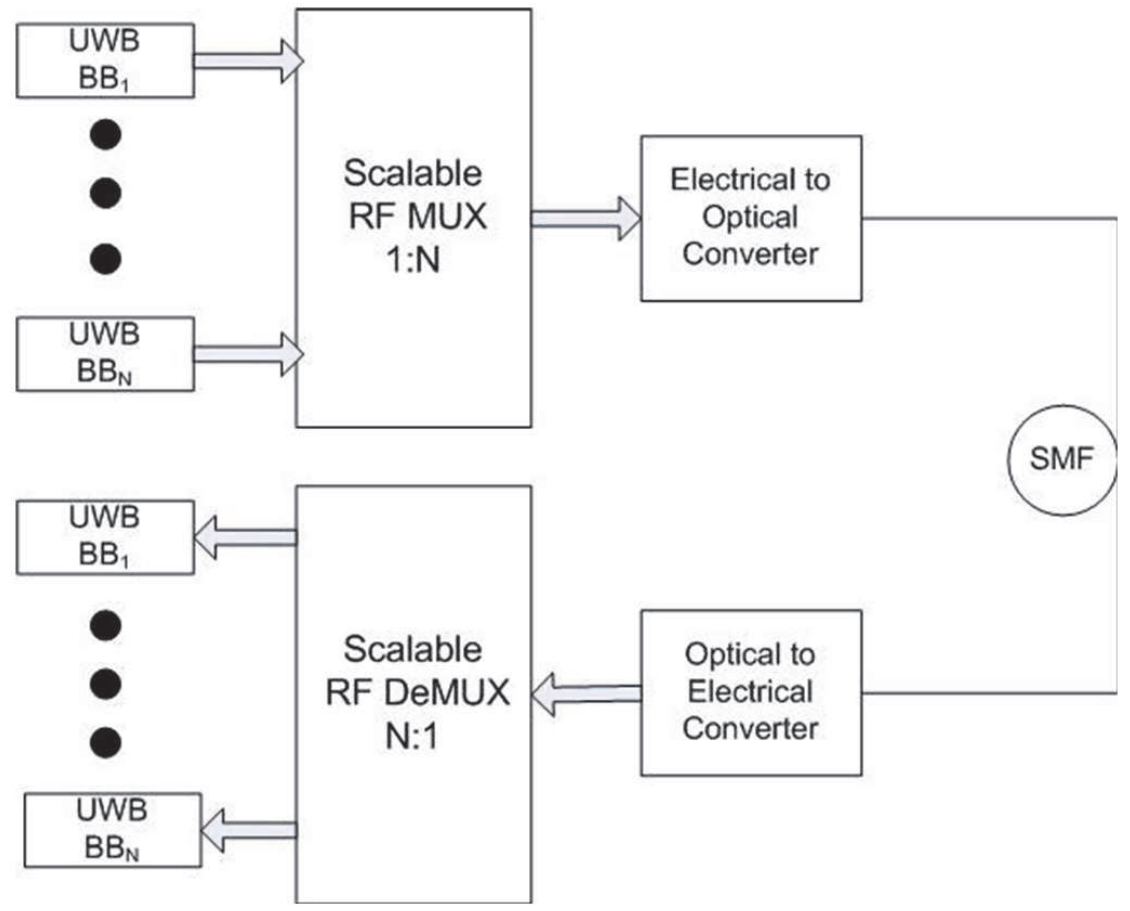

Fig. 12. $61.44 \mathrm{~Gb} / \mathrm{s}$ based on parallel RF/Serial optics with $\mathrm{N}=128$ channels

Another architecture based on parallel-RF/parallel-optics based on 12 low cost VCSELs array that transmits over bus of $12 \mathrm{MMF}$ fibers to array of 12 photo detectors $\mathrm{s}$ is analysed in Ben-Ezra (2010).

\subsection{MB OFDM UWB with MIMO over the wireless channel}

Dense deployment of low-power femto BSs offers significantly higher capacity per area than legacy macrocells due to more efficient spatial re-used and smaller cell size. MIMO techniques permit to exploit the rich scattering of short range indoor wireless channel to increase the spectral efficiency linearly. The UWB-MIMO studies can be classified into four categories Kaiser (2009):

- MIMO channel modelling and measurement. Spatial characterization of UWB MIMO channel is given in Malik (2008).

- Channel capacity. It was shown in Zheng (2006) and Martini (2007) that the system capacity is limited by $N_{T}$, the number of Tx antennas, $N_{R}$ number of receiving antennas, and $\eta$, the number of spatial degrees of freedom of scattered field. A fundamental result by Telatar (2000) showed that for achieving channel capacity at low SNR input signals must be spiky in time or frequency.

- $\quad$ Space-Time Coding (STC) for MB OFDM MIMO is presented in Siriwongpairat (2006). By applying space-time-frequency coding across K OFDM symbols maximum achievable diversity order of $K L N_{T} N_{R}$ can be achieved, where $L$ is the number of resolvable paths. 
- Beam forming for MB OFDM UWB is presented in Malik (2006). It is shown that the signal bandwidth has little impact on beam width or direction

The high level scenario of MB-OFDM UWB with MIMO processing over the wireless channel is shown in Fig. 13. It is assumed that HAN nodes are separated 10m apart from each other, and a latency control to support the MIMO algorithm will be needed. Each cluster should support 10-100 femtocells. The concentrator achieves macro diversity

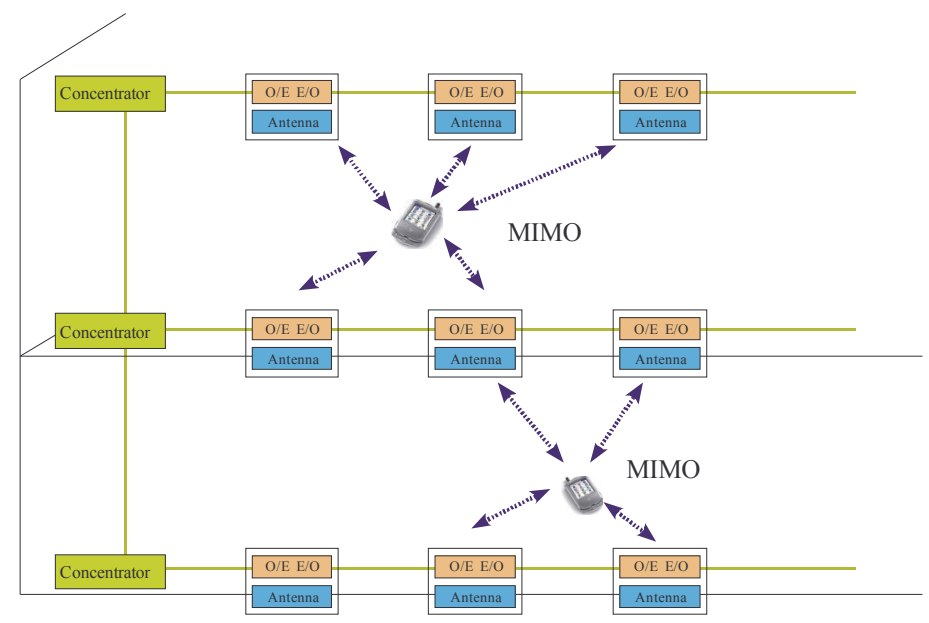

Fig. 13. Multimcell BS processing with MIMO over the air.

\subsection{MB OFDM UWB with MIMO over the wireless-MMF channel}

Recently, the MIMO approach to exploit the modal dispersion in MMF has been address by several researchers Targihat (2007), Greenberg (2007), Greenberg (2008), Armstrong (2009), and Shieh (2010).

The application of MIMO processing to the hybrid wireless-MMF may provide solution for implementing the VLR-DAS paradigm and approaching the challenging goal of green femtocell. The proposed approach paves the way for a green framework in which increase of $x 100$ in energy efficiency and x100 reduction of human exposure to wireless radiation become feasible.

The fundamentals and challenges of this hybrid wireless-optical are yet to be explored.

\section{Conclusion and further prospective}

The research on UWB-MIMO is still in its early stage. Further studies, especially on its MB OFDM implementation, are necessary to bring this technology into the market. The research on MB OFDM UWB over fiber-wireless channels with MIMO presents a promising solution for achieving data rates needed for $4 \mathrm{G}$ indoor systems at extremely low power.

\section{Acknowledgment}

The authors would like to thank the partners of HOMESNET project (CELTIC CP6-009) for the helpful discussions on future femtocells technologies and trends. 


\section{References}

Altman, Z.; Balageas, C.; Formet, E.; Hämäläinen, J.; Marce, O. ; Mutafungwa, E.; Z Zheng, Z Z.; Ran, M.; Ben-Ezra, Y.; Beltran, P. \& Perales S. (2010). Femtocells: The HOMESNET Vision, 21st Annual IEEE Int'l. Symposium on Personal, Indoor and Mobile Radio Communications (PIMRC 2010), Istanbul, Turkey, 26-29 September 2010

Armstrong, J. (2009), OFDM for optical communications, J. Lightwave Technology Vol. 27 N.o.3 pp.189-204

Ben-Ezra, Y.; Ran, M.; Lembrikov, B.I.; Mahlab, U; Haridim, M. \& Leibovich, A. (2010). Optimal transmission of OFDM ultra-wideband signals beyond $40 \mathrm{~Gb} / \mathrm{s}$, Special issue on transparent optical networking, J. of Networks, Vol. 5, No.2, (February 2010), pp. 140-151 [Invited]

CELTIC Purple Book by CELTIC-Plus Version 2011, http://ebookbrowse.com/celtic-pluspurple-book-2011-web-pdf-d110054593

Chow, P; Karim, A; Fung, V \& Dietrich C. (1994). Performance advantages of distributed antennas in indoor wireless communications systems. IEEE VT conference, vol.3 pp. 1522-1526, June 1994.

ECMA-368 http://www.ecma-international.org/publications/standards/Ecma-368.htm

ECMA-387 http://www.ecma-international.org/publications/standards/Ecma-387.htm

FCC 2002 www.fcc.gov/Speeches/misc/statements/knapp060502.pdf

Foschini, G. J.; Karakayali, K. R. \& Valenzuela, A. (2006). Coordinating multiple antenna cellular networks to achieve enormous spectral efficiency, Communications, IEE Proceedings, vol. 153, no. 4, (August 2006), pp. 548 - 555.

IEEE802.16-2009 IEEE Std. 802.16-2009, IEEE Standard for Local and Metropolitan area network part 16: Air Interface for Broadband wireless access systems, May 29, 2009.

Gambini, J. \& Spagnolini, U. (2010). Radio over telephone lines in femtocell systems, in Proc.IEEE 21st International Symposium on Personal Indoor and Mobile Radio Communications (PIMRC, Sept. 2010), pp. 1544-1549.

Gidding, R.P.; Jin, X.O. \& Tang, J.M. (2009). First experimental demonstration of 6Gb/s realtime OFDM transceivers incorporating chanel estimation and variable power loading. Opt. Express Vol. 17 No. 2 pp. 19727-19738.

Greenberg, M.; Nazarathy M. \& Orensein M. (2007). Data parallelization by optical MIMO transmission over multimode fiber with intermodal coupling. J. of lightwave technology vol.25, no.6 (June 2007), pp.1503-1514.

Greenberg, M.; Nazarathy M. \& Orensein M. (2008). Performance of high-bitrate multipleoutput links over multimode fiber with intermodal dispersion J. of lightwave technology Vol.26, No.14 (July 2008), pp.2192-2200.

Heath, R.W; Wu, T; Kwon, Y.H \& Soong, A.C.K (2010). Multiuser MIMO in Distributed Antenna System. Signals, Systems and Computers, Asilomar 2010.

Hillerkuss D. et al., (2010), OFDM for optical communications, OPTICS EXPRESS, vol.18, No.9 (April 2010), pp. 9324-9340.

Jarich, P. (2010) Femtocells Circa 2011: Surveying the Ecosystem Players, in www.currentanalysis.com

Kaiser, T.; Zheng, F. \& Dimitinov, E (2009). An overview of ultra wideband systems with MIMO. Proceedings of the IEEE, Vol. 97, No.2 February 2009, pp.285-312.

Kraemer, R and Katz, M.D (editors) (2009), Short-Range wireless communications Emerging Technologies and Applications, Chapter 24, pp274-327: Ultra-Widedband Radio-Over-Optical Fiber Technologies. Wiley-WWRF series ISBN 978-0-47-69995-9 
Li, X; Luo, M et al. Downlink performance and capacity of distributed antenna system in multi-user scenario, in Wicom, 2009 pp1-4.

Malik, W.Q (2008) Spatial correlation in UWB channels. IEEE Transaction on Wireless communications vol. 7 no. 2, 604-610.

Malik, W.Q.; Allen, B. \& Eswards, D.J. (2006), A simple adaptive beam former for UWB wireless systems, in IEEE conferece on ultra-wideband, pp.453-457.

Martini, A. et al. (2007). Capacity of wideband MIMO channels via Space Time diversity of scattered fields, in IEEE Asilomar Conference on Signals, Systems and Computers pp.138-142.

Perez, D.L.; Valcarce, A.; De La Roche, G. \& Zhang, J. (2009). OFDMA Femtocells: a roadmap on interference avoidance. IEEE Comm. Magazine (Sep 2009), pp41-48

Rappaport, T. S. (2002). Wireless Communications Principles and Practice, Prentice Hall PTR, ISBN 0-13-042232-0, New Jersey, USA

Ran, M.; Lembrikov, B.I. \& Ben-Ezra, Y. (2010a). Ultra-Wideband Radio-Over-Optical Fiber Concepts, Technologies and Applications, IEEE Photonics Journal, Vol. 2, No.1 (February 2010), pp. 36-48, [Invited]

Ran, M.; Ben-Ezra, Y. \& Lembrikov, B.I. (2010b). High Performance Analog Optical Links Based on quantum dot devices for UWB signal transmission, in: in: Ultra WidebandBoris Lembrikov (ed.) Sciyo, Croatia, 2010, pp. 75-96, ISBN 978-953-307-139-8

Ran, M.; Lebovitch, A.; Yurchenko, Y. \& Ben-Ezra Y. (2010c). Green Femtocell: The VLRDAS Approach, 2nd Int'l. Conf. on Evolving Internet (INTERNET 2010), pp. 226-228 Valencia, Spain, September 20-25, 2010

Ran, M; Ben Ezra, Y. \& Lembrikov B.I. (2009). Ultra-wideband Radio-over-optical-fiber Technologies, In: Short-Range Wireless Communications, Kraemer, R. \& Katz, M. D. (Eds.), pp.271-327,Wiley, ISBN 978-0-470-69995-9 (H/B), Chichester, England

RFC3261, SIP: Session Initiation Protocol, IETF, June 2002

Roche, G.; Valcarce, A.; Lopez-Perez, D. \& Zhang, J. (2010). Access Control Mechanisms for Femtocells, IEEE Comm. Magazine, (January 2010), pp. 33-39

Saleh, A.A.M; Rustako, A.J. Jr \& Roman, R.S. (1987). Distributed antennas for indoor radio communications. IEEE Trans. on Communications, vol. 35 no. 12, pp.1245-1251.

Saleh, A and Valenzuela, R.A (1987a). A statistical model for indoor multipath propogation, IEEE Journal on selected Areas Communications, Vol. 5, No.2 pp.128-137

Siriwongpairat, W.P. et al. Multiband-OFDM MIMO coding framework for UWB communication system. IEEE transaction on Signals Processing Vol. 54. No. 4 pp. 214-224.

Tarighat, A. et. al (2007). Fundamental and challenges of optical MIMO multimode fiber links. IEEE communications Magazine, May 2007, pp.57-63

Telatar, I.E. and Tse, D.N.C (2000). Capacity and mutual information of wideband multipath fading channels. IEEE trans. On Information Theory vol. 46, No. 4 pp.1384-1400.

TS23.238, 3GPP (2006) Technical Specification Group Services and Systems Aspects, IP Multimedia Subsystem (IMS), Stage 2, VS.15.0

[UROOF] http://www.ist-uroof.org/

Yongho, R.; Kwak. J.S. \& Etemad, K., (2009). WiMax Femtocell: Requirements, Challenges, and Solutions, IEEE communication Magazine, (September 2009), pp.84-91

Zheng, F. and Kaiser T. (2006) Channel capacity of MIMO UWB indoor wireless systems, in UWB Communication Systems - A comprehensive overview. Hindawi Publishing Corp. pp.376-409.

Zhang, J. \& De la Roche, G. (2010). Femtocells: technologies and deployment, John Wiley \& Sons Ltd., ISBN 978-0-470-74298-3 


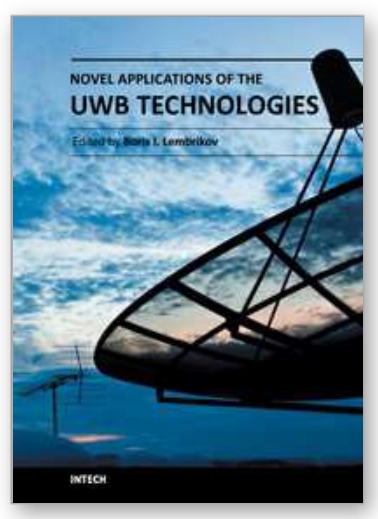

\author{
Novel Applications of the UWB Technologies \\ Edited by Dr. Boris Lembrikov
}

ISBN 978-953-307-324-8

Hard cover, 440 pages

Publisher InTech

Published online 01, August, 2011

Published in print edition August, 2011

Ultra wideband (UWB) communication systems are characterized by high data rates, low cost, multipath immunity, and low power transmission. In 2002, the Federal Communication Commission (FCC) legalized low power UWB emission between $3.1 \mathrm{GHz}$ and $10.6 \mathrm{GHz}$ for indoor communication devices stimulating rapid development of UWB technologies and applications. The proposed book Novel Applications of the UWB Technologies consists of 5 parts and 20 chapters concerning the general problems of UWB communication systems, and novel UWB applications in personal area networks (PANs), medicine, radars and localization systems. The book will be interesting for engineers and researchers occupied in the field of UWB technology.

\title{
How to reference
}

In order to correctly reference this scholarly work, feel free to copy and paste the following:

Moshe Ran and Yossef Ben Ezra (2011). Green Femtocell Based on UWB Technologies, Novel Applications of the UWB Technologies, Dr. Boris Lembrikov (Ed.), ISBN: 978-953-307-324-8, InTech, Available from: http://www.intechopen.com/books/novel-applications-of-the-uwb-technologies/green-femtocell-based-on-uwbtechnologies

\section{INTECH}

open science | open minds

\section{InTech Europe}

University Campus STeP Ri Slavka Krautzeka 83/A 51000 Rijeka, Croatia Phone: +385 (51) 770447 Fax: +385 (51) 686166 www.intechopen.com

\section{InTech China}

Unit 405, Office Block, Hotel Equatorial Shanghai No.65, Yan An Road (West), Shanghai, 200040, China 中国上海市延安西路65号上海国际贵都大饭店办公楼405单元 Phone: +86-21-62489820

Fax: +86-21-62489821 
(C) 2011 The Author(s). Licensee IntechOpen. This chapter is distributed under the terms of the Creative Commons Attribution-NonCommercialShareAlike-3.0 License, which permits use, distribution and reproduction for non-commercial purposes, provided the original is properly cited and derivative works building on this content are distributed under the same license. 